Kompass

Neumología

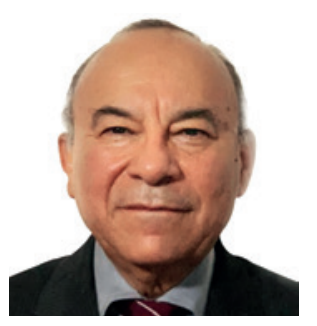

Francisco Espinosa-Larrañaga

Coeditor

Hacia el año 620, Isidro de Sevilla ( 560636) recopila la ciencia de principios de la Edad Media. En el cuarto libro de su obra «De medicina» formula la triada clásica de las medidas terapéuticas que el médico debe aplicar:

- La diaeta, el modo de vida sano

- La pharmacia, la doctrina de los medicamentos

- La chirugia, la intervención quirúrgica Isidro de Sevilla destaca dos tareas principales: la profilaxis y la terapia, está última a la vez la divide en medicina interna y práctica quirúrgica. Es aquí donde se menciona por primera vez a la medicina interna como una forma de tratar a las enfermedades sin la aplicación de técnica quirúrgicas.

El médico practicante de la medicina interna tenía tres propósitos al atender un paciente: reconocer el pasado, a través de la anamnesis con la elaboración de la historia de la enfermedad; conocer el presente del padecimiento, através de la exploración física del enfermo y sus manifestaciones clínicas; y por último identificar un posible futuro con la identificación y pronunciamiento de un pronóstico.

El 6 de enero de 1536 fue inaugurado en la Nueva España el Imperial Colegio de la Santa Cruz de Tlatelolco. Contaba entre otras cátedras con la de medicina. Durante la época colonial en México, existían dos establecimientos encargados de la formación de los médicos: La Facultad de Medicina de la Universidad y el Colegio de Cirugía, una

Kompass Neumol 2021;3:1-2

DOI: 10.1159/000515861

\title{
La medicina interna en la historia
}

encaminada a la formación en el arte de la medicina y el otro a las habilidades quirúrgicas como base del tratamiento. En 1580, el médico holandés Pieter van Foreest (15211597) publica su obra «Observationes» una colección de casos clínicos en 32 volúmenes de experiencias con enfermos para el aprendizaje de la medicina. Una serie de propuestas se desarrollan a partir de este momento para revolucionar la medicina como la «extracción de los humores» como una estrategia quirúrgica basada en la patología humoral de Galeno, y la Teoría circulatoria mecánico-matemática de Harvey entre otras. En 1636, en Leiden, médico Hermannus Boerhaave (1668-1723) introduce la enseñanza de la clínica junto a la cama del paciente con lo que se sitúa, uno de los postulados de la medicina interna, la atención del paciente junto a su cama.

Este breve recorrido a través de la historia nos permite identificar el papel que juega la medicina interna en la clínica, el diagnóstico, su instrumentación y su visión de estudio integrador de las enfermedades.

El médico y anatomista italiano Marcello Malpighi (1628-1694) publicó en 1681: «De pulmonibus», un estudio microscópico de los pulmones, convirtiendo a los pulmones en parte central del estudio clínico de los enfermos. También en este año como consecuencia de una evidencia médico-judicial de la flotación de los pulmones, para deter- minar el momento de la muerte de un recién nacido postuló: «Si el niño respiró fuera de la madre, el pulmón está lleno de aire y flota en el agua, Si no flota murió antes del nacer».

En 1683, Thomas Sydenham (1624-1689), médico londinense describe de acuerdo a sus propias observaciones la sintomatología del ataque agudo de gota, así como el diagnóstico diferencial entre reumatismo agudo y gota. Una característica inherente a la práctica de la clínica y del médico internista es la habilidad desarrollada para realizar diagnóstico diferencial entre las enfermedades.

El clérigo e investigador británico Stephen Hales (1677-1761) realiza por primera vez en un animal una medición exacta de la presión sanguínea, sin embargo, fue hasta 1733 cuando publica los resultados de sus extensos experimentos en «Statistical Essays: containing Haemastatis». Instrumentación actualmente vigente en la actividad médica cotidiana.

El catedrático en medicina Johann Juncker (1679-1759) de Halle en 1736, introduce de manera sistemática en la formación de los médicos, la enseñanza clínica. Hoy en día sigue siendo la piedra angular en la formación de médicos y de internistas.

Leopold Auenbrugger (1722-1809) médico de origen vienés describe la percusión golpeando el tórax para descubrir y diagnosticar enfermedades ocultas en 1761, sin embargo la comunidad médica no le dio im- 
portancia a la técnica de percusión hasta que el Jean Nicolás Corvisart (1755-1821) traduce en el año de 1808 en Paris, la obra de Auenbrugger y consigue que sea parte básica de la exploración física de los pacientes.

El auge que el sistema respiratorio y los pulmones tenían entre los médicos, también fue motivo de creatividad y desarrollo técnico, cuando en 1819, René Théophile Hyacinthe Laennec (1781-1826) publica su obra principal sobre la auscultación mediata o tratado sobre el diagnóstico de las enfermedades pulmonares y cardíacas basado en la exploración con ayuda del estetoscopio.

El descubrimiento del estetoscopio permitió la auscultación del cuerpo de los enfermos y dio lugar a la técnicas predominantes de exploración física realizadas por los médicos internistas, aún hoy en día: la inspección (observación), la palpación, la percusión y la auscultación.

En México en el año de 1833 se identifica la necesidad de modernizar la enseñanza de la medicina para cuyo fin se creó el Establecimiento de Ciencias Médicas que tuvo como finalidad una reforma de la enseñan- za de la medicina que integró en una sola carrera la medicina y la cirugía de acuerdo al modelo adoptado en Francia en tiempos de su revolución. Después de la clausura de la Sorbona fueron abiertas instituciones llamadas Écoles de Santé con enseñanza de la medicina y la cirugía en un solo curriculum, a partir de textos más modernos y con prácticas junto a la cama del paciente. En México, las cátedras a impartirse fueron anatomía general, descriptiva y patológica, fisiología e higiene, primera y segunda de patología interna, primera y segunda de patología externa, materia médica, primera y segunda de clínica interna, primera y segunda de clínica externa, operaciones y obstetricia, medicina legal y farmacia teórica y práctica.

En 1882 Robert Koch (1843-1910) da a conocer en Berlín el descubrimiento del bacilo de la tuberculosis. Agente etiológico de la tuberculosis enfermedad predominante en la época. El 20 de abril del mismo 1882 se reúne por primera vez en la ciudad alemana de Wiesbaden, el Congreso de Medicina Interna que dará origen a la Primera asociación de Medicina Interna (denominado a partir de 1920 como Sociedad Alemana de
Medicina Interna). En el discurso inaugural de su primer presidente el científico Friedrich Theodor Frerichs (1819-1885), se refleja el estado de la medicina de la época, caracterizado por la progresiva especialización en todos su campos y derivaciones. También menciona que el conocimiento médico ha crecido tanto, que nadie puede ya abarcar y dominar la totalidad en cada una de sus partes, y finalmente conmina a no ignorar el reverso de la moneda: alejarse de la idea unitaria del organismo humano tal como lo concibe la medicina interna para finalmente proponer que la medicina interna realice una función integradora en el enfermo.

Este breve recorrido a través de la historia nos permite identificar el papel que juega la medicina interna en la clínica, el diagnóstico, su instrumentación y su visión de estudio integrador de las enfermedades.

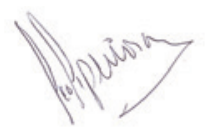

Dr. Francisco Espinosa-Larrañaga Coeditor 